\title{
Optimal Feeding Strategy on Microalgae Growth in Fed-Batch Bioreactor Model
}

\author{
Nailul Izzati and Mardlijah
}

\begin{abstract}
Some countries in the world turn to alternative energy source to fulfill their necessity of fuel. One of the alternative fuels is biodiesel. Raw material of biodiesel can be produced by microalgae cultivation in fed-batch bioreactor. To improve the productivity of microalgae cultivation, we need to determine the optimal control of microalgae growth. This paper discusses mathematical model of microalgae growth in fed-batch bioreactor, and solves the optimal feeding strategy problem by using Pontryagin Minimum Principle. Then we compare the controlled microalgae growth model with the uncontrolled one. Numerical simulation with DOTcvpSB shows that the controlled microalgae growth model yields more harvest and less cost function than the uncontrolled one.
\end{abstract}

Index Terms-Bioreactor model, optimal control.

\section{INTRODUCTION}

B IODESEL is one of the alternative energy sources that becomes popular in recent decades. Biodesel can be produced from microalgae biomass. Microalgae as one of biomass sources, has many advantages, such as can be cultivated throughout the year, need less water and field. Microalgae can grow fast in rich oil content. Oil content of microalgae can reach $80 \%$ of its dry biomass weight. Oil content that produced from microalgae cultivation depends on microalgae growth rate and oil content in its biomass [1].

De la Hoz Siegler et al. [2] constructed a mathematical model to describe microalgae growth in fed-batch bioreactor. Then the optimization of the model to find the optimal feeding strategy is solved by using adaptive model predictive control [3]. The optimal control problem of the model is also discussed by Abdollahi and Dubljevic [4]. They solved the optimal control problem to optimize biomass and lipid productivity by using Interior Point Optimization (IPOPT), Model Predictive Control (MPC), and Moving Horizon Estimation (MHE).

The model constructed in [2] assumes glycine, glucose, biomass, lipid, nitrogen concentration, and bioreactor volume as state variables. In this paper, we discuss a modification of the model and then solve the optimal control problem to optimize biomass and lipid productivity and microalgae feeding cost by using Pontryagin Minimum Principle.

\section{Mathematical Model of Microalgae Growth in FED-BATCH BIOREACTOR}

The dynamic of microalgae growth in fed-batch bioreactor is modeled by De la Hoz Siegler et al. [2] by (1)-(6).

Manuscript received December 23, 2015; accepted January 20, 2016.

The authors are with the Department of Mathematics, Institut Teknologi Sepuluh Nopember, Surabaya 60111, Indonesia. Emails: nellizz89@yahoo.com, mardlijah@gmail.com
System (1)-(6) describes biomass and lipid production of microalgae Auxenochlorella protothecoides. Microalgae growth in bioreactor needs some substrates as nutrition sources. In this model, De la Hoz Siegler et al. use two substrates, i.e. glycine as nitrogen source, and glucose as carbon source. The model assumes that the microalgae cell contains three components, i.e. active biomass, lipid, and nitrogen. These three components can convert from one component to another component with constant yields $Y_{i / j}$.

$$
\begin{aligned}
\frac{d S_{1}}{d t} & =-\rho x+s_{1}^{i} \frac{f_{1}^{i}}{V}-S_{1} D \\
\frac{d S_{2}}{d t} & =\frac{-1}{Y_{x / s}} \mu x-\frac{1}{Y_{p / s}} \pi x-k_{m} x+s_{2}^{i} \frac{f_{2}^{i}}{V}-S_{2} D \\
\frac{d x}{d t} & =\mu x-x D \\
\frac{d p}{d t} & =\pi x-\frac{1}{Y_{x / p}} \mu x-p D \\
\frac{d q}{d t} & =\rho x-\frac{1}{Y_{x / q}} \mu x-q D \\
\frac{d V}{d t} & =V D-f^{o}
\end{aligned}
$$

The model consider six state variables, they are $S_{1}, S_{2}, x$, $p, q, V$ that represent glycine, glucose, biomass, lipid, and nitrogen concentration in fed-batch bioreactor, and volume of the bioreactor, respectively. $\rho$ is the nitrogen source uptake rate, $\pi$ is the lipid production rate, and $\mu$ is the microalgae growth rate. $s_{1}^{i}$ and $s_{2}^{i}$ are the concentration of feeding nutrition. $f_{1}^{i}$ and $f_{2}^{i}$ are the feeding nutrition rate. $f^{o}$ is the outflow, $k_{m}$ is the maintenance factor, and $D=\left(f_{1}^{i}+f_{2}^{i}\right) / V$ is the dilution rate.

\section{METHODS}

This section discusses the methods used in this study. Firstly, we modify the model by using different assumption of variable state and parameter. Then we discuss the optimal control problem and solve it by using Pontryagin Minimum Principle and DOTcvpSB.

\section{A. Modification of The Mathematical Model}

In this study, the volume of fed-batch bioreactor is assumed as a constant parameter. So, we only consider $S_{1}, S_{2}, x, p$, $q$ as the state variables of the model. We also assume the nitrogen source uptake rate, the lipid production rate, and the microalgae growth rate as (7)-(9). Where $\rho_{\max }$ is the maximal nitrogen source uptake rate, $\pi_{\max }$ is the maximal 
lipid production rate, $\mu_{\max }$ is the maximal microalgae growth rate, and

$$
\begin{aligned}
\mu & =\mu_{\max } \frac{S_{2}}{K_{S_{2}}+S_{2}} \frac{q}{K_{q}+q} \\
\pi & =\pi_{\max } \frac{S_{2}}{K_{S_{2}}+S_{2}} \\
\rho & =\rho_{\max } \frac{S_{1}}{K_{S_{1}}+S_{1}}
\end{aligned}
$$

$K_{S_{1}}, K_{S_{2}}, K_{q}$ are the half saturation constant of glycine, glucose, and nitrogen concentration, respectively.

\section{B. Pontryagin Minimum Principle}

Optimal control problem consists of three main components, i.e. mathematical model, objective function, and boundary conditions and physical constrains of state/control variables. Let the mathematical model be given by

$$
\dot{\mathbf{x}}(t)=\mathbf{f}(\mathbf{x}(t), \mathbf{u}(t), t),
$$

and objective function

$$
J=S\left(\mathbf{x}\left(t_{f}\right), t_{f}\right)+\int_{t_{0}}^{t_{f}} \phi(\mathbf{x}(t), \mathbf{u}(t), t) d t,
$$

with boundary conditions

$$
\mathbf{x}\left(t_{0}=0\right)=\mathbf{x}_{0} \quad \text { and } \quad \mathbf{x}\left(t_{f}\right)=\mathbf{x}_{f},
$$

and physical constraints

$$
\mathbf{U}_{-} \leq \mathbf{u}(t) \leq \mathbf{U}_{+} \quad \text { and } \quad \mathbf{X}_{-} \leq \mathbf{x}(t) \leq \mathbf{X}_{+} .
$$

To solve the optimal control problem with Pontryagin Minimum Principle, we need to do the steps as follows [5]:

1) Form the Hamiltonian function.

$$
H(\mathbf{x}, \mathbf{u}, \lambda(t), t)=\phi(\mathbf{x}(t), \mathbf{u}(t), t)+\lambda^{\prime}(t) \mathbf{f}(\mathbf{x}(t), \mathbf{u}(t), t)
$$

2) Find the optimal control $\mathbf{u}^{*}(t)=\mathbf{h}\left(\mathbf{x}^{*}(t), \lambda^{*}(t), t\right)$ by minimizing $H$ w.r.t. $\mathbf{u}(t)$.

$$
\left(\frac{\partial H}{\partial \mathbf{u}}\right)_{*}=0
$$

3) Substitute the optimal control to Hamiltonian function.

$$
H^{*}\left(\mathbf{x}^{*}(t), \mathbf{h}\left(\mathbf{x}^{*}(t), \lambda^{*}(t), t\right), \lambda^{*}(t), t\right)
$$

4) Solve the state and costate differential equations

$$
\dot{\mathbf{x}}^{*}(t)=\left(\frac{\partial H}{\partial \lambda}\right)_{*} \text { and } \quad \dot{\lambda}^{*}(t)=-\left(\frac{\partial H}{\partial \mathbf{x}}\right)_{*}
$$

with initial and final conditions

$$
\left(H+\frac{\partial S}{\partial t}\right)_{*_{t_{f}}} \delta t_{f}+\left(\left(\frac{\partial S}{\partial \mathbf{x}}\right)-\lambda(t)\right)_{* t_{f}} \delta \mathbf{x}_{f}=0 .
$$

\section{DOTcvpSB}

DOTcvpSB is a toolbox of MATLAB that can be used to solve an optimal control problem numerically. DOTcvpSB focuses on system biology problems. To solve an optimal control problem with this toolbox, we need to define the ordinary differential equations that describe the system, the objective function, initial and terminal time problems, constraints of the variables, initial and terminal conditions. This toolbox also allows us to check the simulation of the systems, which only shows the dynamics of the systems without optimization [6].

\section{DISCUSSION AND RESULTS}

\section{A. Mathematical Model}

Consider the assumption explanation in the method section, we can express the mathematical model of microalgae growth in fed-batch bioraector as (10)-(14).

$$
\begin{aligned}
& \frac{d S_{1}}{d t}=-\rho_{\max } \frac{S_{1}}{K_{S_{1}}+S_{1}} x+s_{1}^{i} \frac{f_{1}^{i}}{V}-S_{1} D \\
& \frac{d S_{2}}{d t}=\left(\frac{-1}{Y_{x / s}} \mu_{\max } \frac{q}{K_{q}+q}-\frac{1}{Y_{p / s}} \pi_{\max }\right) \frac{S_{2}}{K_{S_{2}}+S_{2}} x \\
& -k_{m} x+s_{2}^{i} \frac{f_{2}^{i}}{V}-S_{2} D, \\
& \frac{d x}{d t}=\mu_{\max } \frac{S_{2}}{K_{S_{2}}+S_{2}} \frac{q}{K_{q}+q} x-x D, \\
& \frac{d p}{d t}=\pi_{\max } \frac{S_{2}}{K_{S_{2}}+S_{2}} x-\frac{1}{Y_{x / p}} \mu_{\max } \frac{S_{2}}{K_{S_{2}}+S_{2}} \frac{q}{K_{q}+q} x \\
& \frac{d q}{d t}=\rho_{\max } \frac{S_{1}}{K_{S_{1}}+S_{1}} x-\frac{1}{Y_{x / q}} \mu_{\max } \frac{S_{2}}{K_{S_{2}}+S_{2}} \frac{q}{K_{q}+q} x \\
& -q D .
\end{aligned}
$$

\section{B. Optimal Control Problem}

The mathematical model in this optimal control problem is defined by (10)-(14), where $f_{1}^{i}$ and $f_{2}^{i}$ are defined as control variables. The aim of this optimal control problem is to maximize biomass and lipid concentration and minimize the feeding cost. Thus, the objective function of the problem can be written as

$$
J=\int_{t_{0}}^{t_{f}}\left(\frac{C_{1}}{2} u_{1}^{2}+\frac{C_{2}}{2} u_{2}^{2}-x-p\right) d t .
$$

The constraint of control variables are $0 \leq u_{1} \leq 2$ and $0 \leq$ $u_{2} \leq 10$. We have applied the Pontryagin Minimum Principle to the above optimal control problem, as follows.

1) Hamiltonian function

$$
\begin{aligned}
H & =-x-p+\frac{C_{1}}{2} u_{1}^{2}+\frac{C_{2}}{2} u_{2}^{2} \\
& +\lambda_{1}\left(-\rho_{\max } \frac{S_{1}}{K_{S_{1}}+S_{1}} x+s_{1}^{i} \frac{u_{1}}{V}-S_{1} \frac{u_{1}+u_{2}}{V}\right) \\
& +\lambda_{2}\left(\frac{-1}{Y_{x / s}} \mu_{\max } \frac{S_{2}}{K_{S_{2}}+S_{2}} \frac{q}{K_{q}+q} x\right. \\
& \left.-\frac{1}{Y_{p / s}} \pi_{\max } \frac{S_{2}}{K_{S_{2}}+S_{2}} x\right) \\
+ & \lambda_{2}\left(-k_{m} x+s_{2}^{i} \frac{u_{2}}{V}-S_{2} \frac{u_{1}+u_{2}}{V}\right) \\
+ & \lambda_{3}\left(\mu_{\max } \frac{S_{2}}{K_{S_{2}}+S_{2}} \frac{q}{K_{q}+q} x-x \frac{u_{1}+u_{2}}{V}\right) \\
+ & \lambda_{4}\left(\pi_{\max } \frac{S_{2}}{K_{S_{2}}+S_{2}} x\right. \\
& \left.-\frac{1}{Y_{x / p}} \mu_{\max } \frac{S_{2}}{K_{S_{2}}+S_{2}} \frac{q}{K_{q}+q} x-p \frac{u_{1}+u_{2}}{V}\right) \\
+ & \lambda_{5}\left(\rho_{\max } \frac{S_{1}}{K_{S_{1}}+S_{1}} x\right.
\end{aligned}
$$




$$
\left.-\frac{1}{Y_{x / q}} \mu_{\max } \frac{S_{2}}{K_{S_{2}}+S_{2}} \frac{q}{K_{q}+q} x-q \frac{u_{1}+u_{2}}{V}\right)
$$

2) Optimal control law

$$
\begin{aligned}
u_{1}^{*}= & \frac{1}{C_{1} V}\left(-\lambda_{1} s_{1}^{i}+\lambda_{1} S_{1}+\lambda_{2} S_{2}+\lambda_{3} x+\lambda_{4} p\right. \\
& \left.+\lambda_{5} q\right) \\
u_{2}^{*}= & \frac{1}{C_{2} V}\left(-\lambda_{2} s_{2}^{i}+\lambda_{1} S_{1}+\lambda_{2} S_{2}+\lambda_{3} x+\lambda_{4} p\right. \\
& \left.+\lambda_{5} q\right)
\end{aligned}
$$

3) Optimal Hamiltonian function

$$
\begin{aligned}
& H^{*}=-x-p-\lambda_{1}\left(\rho_{\max } \frac{S_{1}}{K_{S_{1}}+S_{1}} x\right) \\
& +\lambda_{2}\left(\frac{-1}{Y_{x / s}} \mu_{\max } \frac{S_{2}}{K_{S_{2}}+S_{2}} \frac{q}{K_{q}+q} x\right. \\
& \left.-\frac{1}{Y_{p / s}} \pi_{\max } \frac{S_{2}}{K_{S_{2}}+S_{2}} x-k_{m} x\right) \\
& +\lambda_{3}\left(\mu_{\max } \frac{S_{2}}{K_{S_{2}}+S_{2}} \frac{q}{K_{q}+q} x\right) \\
& +\lambda_{4}\left(\pi_{\max } \frac{S_{2}}{K_{S_{2}}+S_{2}} x\right. \\
& \left.-\frac{1}{Y_{x / p}} \mu_{\max } \frac{S_{2}}{K_{S_{2}}+S_{2}} \frac{q}{K_{q}+q} x\right) \\
& +\lambda_{5}\left(\rho_{\max } \frac{S_{1}}{K_{S_{1}}+S_{1}} x\right. \\
& \left.-\frac{1}{Y_{x / q}} \mu_{\max } \frac{S_{2}}{K_{S_{2}}+S_{2}} \frac{q}{K_{q}+q} x\right) \\
& -\frac{\lambda_{1}^{2}\left(s_{1}^{i}\right)^{2}}{2 C_{1} V^{2}}-\frac{\lambda_{1}^{2} S_{1}^{2}}{2 C_{1} V^{2}}-\frac{\lambda_{2}^{2} S_{2}^{2}}{2 C_{1} V^{2}}-\frac{\lambda_{3}^{2} x^{2}}{2 C_{1} V^{2}} \\
& -\frac{\lambda_{2}^{2}\left(s_{2}^{i}\right)^{2}}{2 C_{2} V^{2}}-\frac{\lambda_{1}^{2} S_{1}^{2}}{2 C_{2} V^{2}}-\frac{\lambda_{2}^{2} S_{2}^{2}}{2 C_{2} V^{2}}-\frac{\lambda_{3}^{2} x^{2}}{2 C_{2} V^{2}} \\
& -\frac{\lambda_{4}^{2} p^{2}}{2 C_{1} V^{2}}-\frac{\lambda_{5}^{2} q^{2}}{2 C_{1} V^{2}}-\frac{\lambda_{4}^{2} p^{2}}{2 C_{2} V^{2}}-\frac{\lambda_{5}^{2} q^{2}}{2 C_{2} V^{2}} \\
& +\frac{\lambda_{1} \lambda_{2} s_{1}^{i} S_{2}}{C_{1} V^{2}}+\frac{\lambda_{1}^{2} S_{1} s_{1}^{i}}{C_{1} V^{2}}+\frac{\lambda_{1} \lambda_{3} s_{1}^{i} x}{C_{1} V^{2}}+\frac{\lambda_{1} \lambda_{4} s_{1}^{i} p}{C_{1} V^{2}} \\
& +\frac{\lambda_{1} \lambda_{5} s_{1}^{i} q}{C_{1} V^{2}}+\frac{\lambda_{1} \lambda_{2} S_{1} s_{2}^{i}}{C_{2} V^{2}}+\frac{\lambda_{2}^{2} S_{2} s_{2}^{i}}{C_{2} V^{2}}+\frac{\lambda_{2} \lambda_{3} s_{2}^{i} x}{C_{2} V^{2}} \\
& +\frac{\lambda_{2} \lambda_{4} s_{2}^{i} p}{C_{2} V^{2}}+\frac{\lambda_{2} \lambda_{5} s_{2}^{i} q}{C_{2} V^{2}}-\frac{\lambda_{1} \lambda_{2} S_{1} S_{2}}{C_{1} V^{2}}-\frac{\lambda_{1} \lambda_{3} S_{1} x}{C_{1} V^{2}} \\
& -\frac{\lambda_{2} \lambda_{3} S_{2} x}{C_{1} V^{2}}-\frac{\lambda_{1} \lambda_{4} S_{1} p}{C_{1} V^{2}}-\frac{\lambda_{2} \lambda_{4} S_{2} p}{C_{1} V^{2}}-\frac{\lambda_{3} \lambda_{4} x p}{C_{1} V^{2}} \\
& -\frac{\lambda_{1} \lambda_{5} S_{1} q}{C_{1} V^{2}}-\frac{\lambda_{2} \lambda_{5} S_{2} q}{C_{1} V^{2}}-\frac{\lambda_{3} \lambda_{5} x q}{C_{1} V^{2}}-\frac{\lambda_{4} \lambda_{5} p q}{C_{1} V^{2}} \\
& -\frac{\lambda_{1} \lambda_{2} S_{1} S_{2}}{C_{2} V^{2}}-\frac{\lambda_{1} \lambda_{3} S_{1} x}{C_{2} V^{2}}-\frac{\lambda_{2} \lambda_{3} S_{2} x}{C_{2} V^{2}}-\frac{\lambda_{1} \lambda_{4} S_{1} p}{C_{2} V^{2}} \\
& -\frac{\lambda_{2} \lambda_{4} S_{2} p}{C_{2} V^{2}}-\frac{\lambda_{3} \lambda_{4} x p}{C_{2} V^{2}}-\frac{\lambda_{1} \lambda_{5} S_{1} q}{C_{2} V^{2}}-\frac{\lambda_{2} \lambda_{5} S_{2} q}{C_{2} V^{2}} \\
& -\frac{\lambda_{3} \lambda_{5} x q}{C_{2} V^{2}}-\frac{\lambda_{4} \lambda_{5} p q}{C_{2} V^{2}}
\end{aligned}
$$

4) State and costate differential equations

$$
\dot{S}_{1}^{*}=-\rho_{\max } \frac{S_{1}}{K_{S_{1}}+S_{1}} x-\frac{\lambda_{1}\left(s_{1}^{i}\right)^{2}}{C_{1} V^{2}}-\frac{\lambda_{1} S_{1}^{2}}{C_{1} V^{2}}-\frac{\lambda_{1} S_{1}^{2}}{C_{2} V^{2}}
$$

$$
\begin{aligned}
& +\frac{\lambda_{2} s_{1}^{i} S_{2}}{C_{1} V^{2}}+\frac{2 \lambda_{1} S_{1} s_{1}^{i}}{C_{1} V^{2}}+\frac{\lambda_{3} s_{1}^{i} x}{C_{1} V^{2}}+\frac{\lambda_{4} s_{1}^{i} p}{C_{1} V^{2}}+\frac{\lambda_{5} s_{1}^{i} q}{C_{1} V^{2}} \\
& +\frac{\lambda_{2} S_{1} s_{2}^{i}}{C_{1} V^{2}}-\frac{\lambda_{2} S_{1} S_{2}}{C_{1} V^{2}}-\frac{\lambda_{3} S_{1} x}{C_{1} V^{2}}-\frac{\lambda_{4} S_{1} p}{C_{1} V^{2}}-\frac{\lambda_{5} S_{1} q}{C_{1} V^{2}} \\
& -\frac{\lambda_{2} S_{1} S_{2}}{C_{2} V^{2}}-\frac{\lambda_{3} S_{1} x}{C_{2} V^{2}}-\frac{\lambda_{4} S_{1} p}{C_{2} V^{2}}-\frac{\lambda_{5} S_{1} q}{C_{2} V^{2}} \\
& \dot{S}_{2}^{*}=\frac{-1}{Y_{x / s}} \mu_{\max } \frac{S_{2}}{K_{S_{2}}+S_{2}} \frac{q}{K_{q}+q} x \\
& -\frac{1}{Y_{p / s}} \pi_{\max } \frac{S_{2}}{K_{S_{2}}+S_{2}} x-k_{m} x-\frac{\lambda_{2} S_{2}^{2}}{C_{1} V^{2}}-\frac{\lambda_{2}\left(s_{2}^{i}\right)^{2}}{C_{2} V^{2}} \\
& -\frac{\lambda_{2} S_{2}^{2}}{C_{2} V^{2}}+\frac{\lambda_{1} s_{1}^{i} S_{2}}{C_{1} V^{2}}+\frac{\lambda_{1} S_{1} s_{2}^{i}}{C_{2} V^{2}}+\frac{2 \lambda_{2} S_{2} s_{2}^{i}}{C_{2} V^{2}}+\frac{\lambda_{3} s_{2}^{i} x}{C_{2} V^{2}} \\
& +\frac{\lambda_{4} s_{2}^{i} p}{C_{2} V^{2}}+\frac{\lambda_{5} s_{2}^{i} q}{C_{2} V^{2}}-\frac{\lambda_{1} S_{1} S_{2}}{C_{1} V^{2}}-\frac{\lambda_{3} S_{2} x}{C_{1} V^{2}}-\frac{\lambda_{4} S_{2} p}{C_{1} V^{2}} \\
& -\frac{\lambda_{5} S_{2} q}{C_{1} V^{2}}-\frac{\lambda_{1} S_{1} S_{2}}{C_{2} V^{2}}-\frac{\lambda_{3} S_{2} x}{C_{2} V^{2}}-\frac{\lambda_{4} S_{2} p}{C_{2} V^{2}}-\frac{\lambda_{5} S_{2} q}{C_{2} V^{2}} \\
& \dot{x}^{*}=\mu_{\max } \frac{S_{2}}{K_{S_{2}}+S_{2}} \frac{q}{K_{q}+q} x-\frac{\lambda_{3} x^{2}}{C_{1} V^{2}}-\frac{\lambda_{3} x^{2}}{C_{2} V^{2}} \\
& +\frac{\lambda_{1} s_{1}^{i} x}{C_{1} V^{2}}+\frac{\lambda_{2} s_{2}^{i} x}{C_{2} V^{2}}-\frac{\lambda_{1} S_{1} x}{C_{1} V^{2}}-\frac{\lambda_{2} S_{2} x}{C_{1} V^{2}}-\frac{\lambda_{4} x p}{C_{1} V^{2}} \\
& -\frac{\lambda_{5} x q}{C_{1} V^{2}}-\frac{\lambda_{1} S_{1} x}{C_{2} V^{2}}-\frac{\lambda_{2} S_{2} x}{C_{2} V^{2}}-\frac{\lambda_{4} x p}{C_{2} V^{2}}-\frac{\lambda_{5} x q}{C_{2} V^{2}} \\
& \dot{p}^{*}=\pi_{\max } \frac{S_{2}}{K_{S_{2}}+S_{2}} x-\frac{1}{Y_{x / p}} \mu_{\max } \frac{S_{2}}{K_{S_{2}}+S_{2}} \frac{q}{K_{q}+q} x \\
& -\frac{\lambda_{4} p^{2}}{C_{1} V^{2}}-\frac{\lambda_{4} p^{2}}{C_{2} V^{2}}+\frac{\lambda_{1} s_{1}^{i} p}{C_{1} V^{2}}+\frac{\lambda_{2} s_{2}^{i} p}{C_{2} V^{2}}-\frac{\lambda_{1} S_{1} p}{C_{1} V^{2}} \\
& -\frac{\lambda_{2} S_{2} p}{C_{1} V^{2}}-\frac{\lambda_{3} x p}{C_{1} V^{2}}-\frac{\lambda_{5} p q}{C_{1} V^{2}}-\frac{\lambda_{1} S_{1} p}{C_{2} V^{2}}-\frac{\lambda_{2} S_{2} p}{C_{2} V^{2}} \\
& -\frac{\lambda_{3} x p}{C_{2} V^{2}}-\frac{\lambda_{5} p q}{C_{2} V^{2}} \\
& \dot{q}^{*}=\rho_{\max } \frac{S_{1}}{K_{S_{1}}+S_{1}} x-\frac{1}{Y_{x / q}} \mu_{\max } \frac{S_{2}}{K_{S_{2}}+S_{2}} \frac{q}{K_{q}+q} x \\
& -\frac{\lambda_{5} q^{2}}{C_{1} V^{2}}-\frac{\lambda_{5} q^{2}}{C_{2} V^{2}}+\frac{\lambda_{1} s_{1}^{i} q}{C_{1} V^{2}}+\frac{\lambda_{2} s_{2}^{i} q}{C_{2} V^{2}}-\frac{\lambda_{1} S_{1} q}{C_{1} V^{2}} \\
& -\frac{\lambda_{2} S_{2} q}{C_{1} V^{2}}-\frac{\lambda_{3} x q}{C_{1} V^{2}}-\frac{\lambda_{4} p q}{C_{1} V^{2}}-\frac{\lambda_{1} S_{1} q}{C_{2} V^{2}}-\frac{\lambda_{2} S_{2} q}{C_{2} V^{2}} \\
& -\frac{\lambda_{3} x q}{C_{2} V^{2}}-\frac{\lambda_{4} p q}{C_{2} V^{2}} \\
& \dot{\lambda}_{1}^{*}=\left(\lambda_{1} \rho_{\max } x-\lambda_{5} \rho_{\max } x\right)\left(\frac{1}{K_{S_{1}}+S_{1}}\right. \\
& \left.-\frac{S_{1}}{\left(K_{S_{1}}+S_{1}\right)^{2}}\right)+\frac{\lambda_{1}^{2} S_{1}}{C_{1} V^{2}}+\frac{\lambda_{1} \lambda_{2} S_{2}}{C_{1} V^{2}}+\frac{\lambda_{1} \lambda_{3} x}{C_{1} V^{2}} \\
& +\frac{\lambda_{1} \lambda_{4} p}{C_{1} V^{2}}+\frac{\lambda_{1} \lambda_{5} q}{C_{1} V^{2}}+\frac{\lambda_{1}^{2} S_{1}}{C_{2} V^{2}}+\frac{\lambda_{1} \lambda_{2} S_{2}}{C_{2} V^{2}}+\frac{\lambda_{1} \lambda_{3} x}{C_{2} V^{2}} \\
& +\frac{\lambda_{1} \lambda_{4} p}{C_{2} V^{2}}+\frac{\lambda_{1} \lambda_{5} q}{C_{2} V^{2}}-\frac{\lambda_{1}^{2} s_{1}^{i}}{C_{1} V^{2}}-\frac{\lambda_{1} \lambda_{2} s_{2}^{i}}{C_{2} V^{2}} \\
& \dot{\lambda}_{2}^{*}=\lambda_{2}\left(\frac{1}{Y_{x / s}} \mu_{\max } \frac{q}{K_{q}+q} x-\frac{1}{Y_{p / s}} \pi_{\max }\right)
\end{aligned}
$$




$$
\begin{aligned}
& \left(\frac{1}{K_{S_{2}}+S_{2}}-\frac{S_{2}}{\left(K_{S_{2}}+S_{2}\right)^{2}}\right) \\
& -\lambda_{3}\left(\mu_{\max } \frac{q}{K_{q}+q} x\right)\left(\frac{1}{K_{S_{2}}+S_{2}}\right. \\
& \left.-\frac{S_{2}}{\left(K_{S_{2}}+S_{2}\right)^{2}}\right) \\
& -\lambda_{4}\left(\pi_{\max } x-\frac{1}{Y_{x / p}} \mu_{\max } \frac{q}{K_{q}+q} x\right)\left(\frac{1}{K_{S_{2}}+S_{2}}\right. \\
& \left.-\frac{S_{2}}{\left(K_{S_{2}}+S_{2}\right)^{2}}\right) \\
& +\lambda_{5}\left(\frac{1}{Y_{x / q}} \mu_{\max } \frac{q}{K_{q}+q} x\right)\left(\frac{1}{K_{S_{2}}+S_{2}}\right. \\
& \left.-\frac{S_{2}}{\left(K_{S_{2}}+S_{2}\right)^{2}}\right) \\
& +\frac{\lambda_{2}^{2} S_{2}}{C_{1} V^{2}}+\frac{\lambda_{2}^{2} S_{2}}{C_{2} V^{2}}-\frac{\lambda_{1} \lambda_{2} s_{1}^{i}}{C_{1} V^{2}}-\frac{\lambda_{2}^{2} s_{2}^{i}}{C_{2} V^{2}}+\frac{\lambda_{1} \lambda_{2} S_{1}}{C_{1} V^{2}} \\
& +\frac{\lambda_{2} \lambda_{3} x}{C_{1} V^{2}}+\frac{\lambda_{2} \lambda_{4} p}{C_{1} V^{2}}+\frac{\lambda_{2} \lambda_{5} q}{C_{1} V^{2}}+\frac{\lambda_{1} \lambda_{2} S_{1}}{C_{2} V^{2}}+\frac{\lambda_{2} \lambda_{3} x}{C_{2} V^{2}} \\
& +\frac{\lambda_{2} \lambda_{4} p}{C_{2} V^{2}}+\frac{\lambda_{2} \lambda_{5} q}{C_{2} V^{2}} \\
& \dot{\lambda}_{3}^{*}=-1+\lambda_{1}\left(\rho_{\max } \frac{S_{1}}{K_{S_{1}}+S_{1}}\right) \\
& -\lambda_{2}\left(\frac{-1}{Y_{x / s}} \mu_{\max } \frac{S_{2}}{K_{S_{2}}+S_{2}} \frac{q}{K_{q}+q}\right. \\
& \left.-\frac{1}{Y_{p / s}} \pi_{\max } \frac{S_{2}}{K_{S_{2}}+S_{2}}-k_{m}\right) \\
& -\lambda_{3}\left(\mu_{\max } \frac{S_{2}}{K_{S_{2}}+S_{2}} \frac{q}{K_{q}+q}\right) \\
& -\lambda_{4}\left(\pi_{\max } \frac{S_{2}}{K_{S_{2}}+S_{2}}\right. \\
& \left.-\frac{1}{Y_{x / p}} \mu_{\max } \frac{S_{2}}{K_{S_{2}}+S_{2}} \frac{q}{K_{q}+q}\right) \\
& -\lambda_{5}\left(\rho_{\max } \frac{S_{1}}{K_{S_{1}}+S_{1}}\right. \\
& \left.-\frac{1}{Y_{x / q}} \mu_{\max } \frac{S_{2}}{K_{S_{2}}+S_{2}} \frac{q}{K_{q}+q}\right) \\
& +\frac{\lambda_{3}^{2} x}{C_{1} V^{2}}+\frac{\lambda_{3}^{2} x}{C_{2} V^{2}}-\frac{\lambda_{1} \lambda_{3} s_{1}^{i}}{C_{1} V^{2}}-\frac{\lambda_{2} \lambda_{3} s_{2}^{i}}{C_{2} V^{2}}+\frac{\lambda_{1} \lambda_{3} S_{1}}{C_{1} V^{2}} \\
& +\frac{\lambda_{2} \lambda_{3} S_{2}}{C_{1} V^{2}}+\frac{\lambda_{3} \lambda_{4} p}{C_{1} V^{2}}+\frac{\lambda_{3} \lambda_{5} q}{C_{1} V^{2}}+\frac{\lambda_{1} \lambda_{3} S_{1}}{C_{2} V^{2}}+\frac{\lambda_{2} \lambda_{3} S_{2}}{C_{2} V^{2}} \\
& +\frac{\lambda_{3} \lambda_{4} p}{C_{2} V^{2}}+\frac{\lambda_{3} \lambda_{5} q}{C_{2} V^{2}} \\
& \dot{\lambda}_{4}^{*}=1+\frac{\lambda_{4}^{2} p}{C_{1} V^{2}}+\frac{\lambda_{4}^{2} p}{C_{2} V^{2}}-\frac{\lambda_{1} \lambda_{4} s_{1}^{i}}{C_{1} V^{2}}-\frac{\lambda_{2} \lambda_{4} s_{2}^{i}}{C_{2} V^{2}} \\
& +\frac{\lambda_{1} \lambda_{4} S_{1}}{C_{1} V^{2}}+\frac{\lambda_{2} \lambda_{4} S_{2}}{C_{1} V^{2}}+\frac{\lambda_{3} \lambda_{4} x}{C_{1} V^{2}}+\frac{\lambda_{4} \lambda_{5} q}{C_{1} V^{2}}+\frac{\lambda_{1} \lambda_{4} S_{1}}{C_{2} V^{2}} \\
& +\frac{\lambda_{2} \lambda_{4} S_{2}}{C_{2} V^{2}}+\frac{\lambda_{3} \lambda_{4} x}{C_{2} V^{2}}+\frac{\lambda_{4} \lambda_{5} q}{C_{2} V^{2}} \\
& \dot{\lambda}_{5}^{*}=\lambda_{2}\left(\frac{1}{Y_{x / s}} \mu_{\max } \frac{S_{2}}{K_{S_{2}}+S_{2}} x\right)\left(\frac{1}{K_{q}+q}\right.
\end{aligned}
$$

$$
\begin{aligned}
& \left.-\frac{q}{\left(K_{q}+q\right)^{2}}\right) \\
- & \lambda_{3}\left(\mu_{\max } \frac{S_{2}}{K_{S_{2}}+S_{2}} x\right)\left(\frac{1}{K_{q}+q}-\frac{q}{\left(K_{q}+q\right)^{2}}\right) \\
+ & \lambda_{4}\left(\frac{1}{Y_{x / p}} \mu_{\max } \frac{S_{2}}{K_{S_{2}}+S_{2}} x\right)\left(\frac{1}{K_{q}+q}\right. \\
& \left.-\frac{q}{\left(K_{q}+q\right)^{2}}\right) \\
+ & \lambda_{5}\left(\frac{1}{Y_{x / q}} \mu_{\max } \frac{S_{2}}{K_{S_{2}}+S_{2}} x\right)\left(\frac{1}{K_{q}+q}\right. \\
& \left.-\frac{q}{\left(K_{q}+q\right)^{2}}\right) \\
+ & \frac{\lambda_{5}^{2} q}{C_{1} V^{2}}+\frac{\lambda_{5}^{2} q}{C_{2} V^{2}}-\frac{\lambda_{1} \lambda_{5} s_{1}^{i}}{C_{1} V^{2}}-\frac{\lambda_{2} \lambda_{5} s_{2}^{i}}{C_{2} V^{2}}+\frac{\lambda_{1} \lambda_{5} S_{1}}{C_{1} V^{2}} \\
+ & \frac{\lambda_{2} \lambda_{5} S_{2}}{C_{1} V^{2}}+\frac{\lambda_{3} \lambda_{5} x}{C_{1} V^{2}}+\frac{\lambda_{4} \lambda_{5} p}{C_{1} V^{2}}+\frac{\lambda_{1} \lambda_{5} S_{1}}{C_{2} V^{2}}+\frac{\lambda_{2} \lambda_{5} S_{2}}{C_{2} V^{2}} \\
+ & \frac{\lambda_{3} \lambda_{5} x}{C_{2} V^{2}}+\frac{\lambda_{4} \lambda_{5} p}{C_{2} V^{2}}
\end{aligned}
$$

where the initial condition is $S_{1}(0)=S_{10}, S_{2}(0)=S_{20}$, $x(0)=x_{0}, p(0)=p_{0}, q(0)=q_{0}$; and the final condition is $\lambda_{1}\left(t_{f}\right)=0, \lambda_{2}\left(t_{f}\right)=0, \lambda_{3}\left(t_{f}\right)=0, \lambda_{4}\left(t_{f}\right)=0$, $\lambda_{5}\left(t_{f}\right)=0$.

However, the solution of state and costate differential equations are hard to find analytically. Thus we use DOTcvpSB to solve this numerically.

\section{Numerical Simulation}

In this section, we discuss the numerical simulations of the model. First, we perform simulation for the model without controlling the feeding strategy. Second, we perform simulation with the optimal control of the model. Then, we compare the results of those simulations. Initial conditions that are used in this numerical simulations are $S_{1}(0)=29.3, S_{2}(0)=0.57$, $x(0)=10, p(0)=2$ and $q(0)=2$. These initial conditions describe the concentration of the state variables at time $t_{0}=0$. Table I shows the values of parameters in the model [4].

TABLE I

Parameters of Microalgae Growth in Fed-Batch Bioreactor MODEL.

\begin{tabular}{|c|c|c|}
\hline Parameter & Value & Unit \\
\hline$Y_{x / s}$ & 0.55 & - \\
$Y_{p / s}$ & 0.34 & - \\
$Y_{x / q}$ & 56.67 & - \\
$Y_{x / p}$ & 11.84 & - \\
$k_{m}$ & 0.19 & L/day \\
$\mu_{\max }$ & 14.18 & L/day \\
$\pi_{\max }$ & 0.50 & L/day \\
$\rho_{\max }$ & 0.93 & L/day \\
$K_{S_{1}}$ & 0.14 & gr/L \\
$K_{S_{2}}$ & 8.45 & gr/L \\
$K_{q}$ & 0.0041 & gr/L \\
$s_{1}^{i}$ & 0.6 & gr/L \\
$s_{2}^{i}$ & 40 & gr/L \\
$V$ & 2.00 & L \\
\hline
\end{tabular}

Figure 1 shows a simulation of the model without controlling the feeding strategy. In this case, the concentration of 
glycine, glucose, biomass, lipid, and nitrogen at the end of cultivation, are $10.08 \mathrm{gr} / \mathrm{L},-0.02 \mathrm{gr} / \mathrm{L}, 8.55 \mathrm{gr} / \mathrm{L}, 1.83 \mathrm{gr} / \mathrm{L}$, and $18.28 \mathrm{gr} / \mathrm{L}$, respectively. Finally the value of objective function is -22.59 .

TABLE II

RESULTS OF THE CONTROLLED MICROALGAE GROWTH MODEL.

\begin{tabular}{|c|c|c|c|c|c|c|}
\hline \multirow{2}{*}{ Weight } & \multicolumn{7}{|c|}{ State variables } \\
\cline { 2 - 7 } & $S_{1}$ & $S_{2}$ & $x$ & $p$ & $q$ & $J$ \\
\hline$C_{1}=1, C_{2}=1$ & 0.100 & 0.10 & 11.49 & 0.81 & 17.18 & -24.88 \\
$C_{1}=1, C_{2}=2$ & 0.099 & 0.10 & 11.47 & 0.82 & 17.27 & -24.61 \\
$C_{1}=2, C_{2}=2$ & 0.100 & 0.10 & 11.49 & 0.81 & 17.18 & -24.61 \\
$C_{1}=2, C_{2}=3$ & 0.099 & 0.13 & 11.67 & 0.78 & 16.82 & -24.20 \\
$C_{1}=3, C_{2}=2$ & 0.099 & 0.10 & 11.49 & 0.81 & 17.18 & -24.59 \\
\hline
\end{tabular}

Figures 2 and 3 show a simulation of optimal control of the model. By controlling the feeding strategy, we obtain the concentration of glycine, glucose, biomass, lipid, and nitrogen at the end of cultivation, which are $0.10 \mathrm{gr} / \mathrm{L}, 0.10 \mathrm{gr} / \mathrm{L}, 11.49$ $\mathrm{gr} / \mathrm{L}, 0.81 \mathrm{gr} / \mathrm{L}$, and $17.18 \mathrm{gr} / \mathrm{L}$, respectively. The value of objective function is -24.88 .

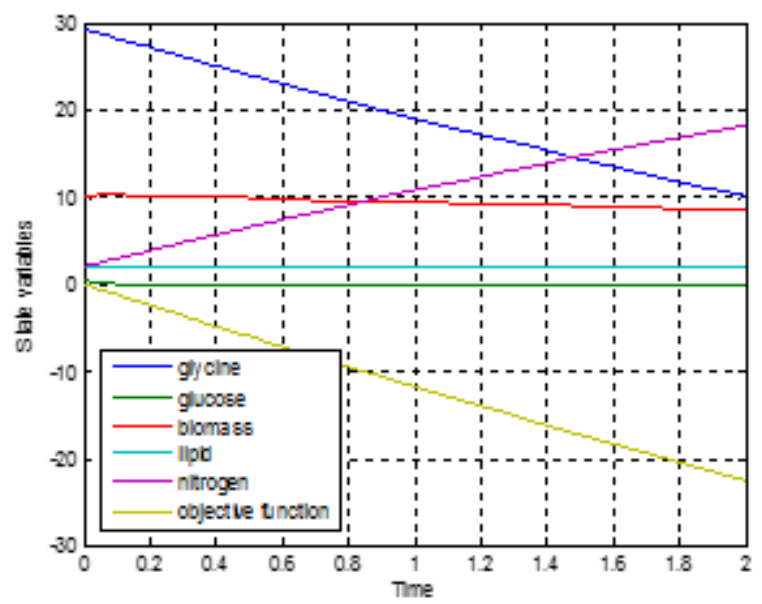

Fig. 1. Numerical solutions of the microalgae growth model without controlling the feeding strategy.

Figure 3 describes the optimal feeding strategy of the model. The feeding rate is adjusted with respect to the problem at hand. The optimal glycine feeding rate is constant 0.1 $\mathrm{mL} /$ day. The optimal glucose feeding rate is decreased from $0.81 \mathrm{~mL} /$ day, $0.71 \mathrm{~mL} /$ day, $0.63 \mathrm{~mL} /$ day, $0.56 \mathrm{~mL} /$ day, 0.50 $\mathrm{mL} /$ day, $0.43 \mathrm{~mL} /$ day, $0.34 \mathrm{~mL} /$ day, to $0.29 \mathrm{~mL} /$ day.

Figures 1-3 are obtained by choosing $C_{1}=1$ and $C_{2}=1$. We present the results of the controlled model for some other values of $C_{1}$ and $C_{2}$ in Table II.

\section{Conclusions}

The solution of optimal control problem of microalgae growth model can be formulated by Pontryagin Minimum Principle, and simulated by DOTcvpSB. However, for some values of $C_{1}$ and $C_{2}$, the numerical simulation shows the decrease of biomass concentration. It implies that the values of control weight on objective function effect the optimization result.

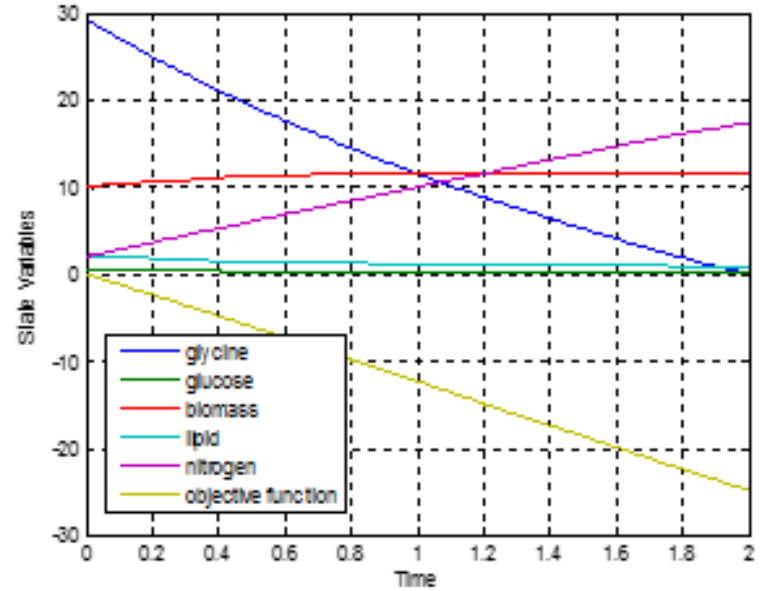

Fig. 2. Numerical solutions of the microalgae growth model with optimal feeding strategy.

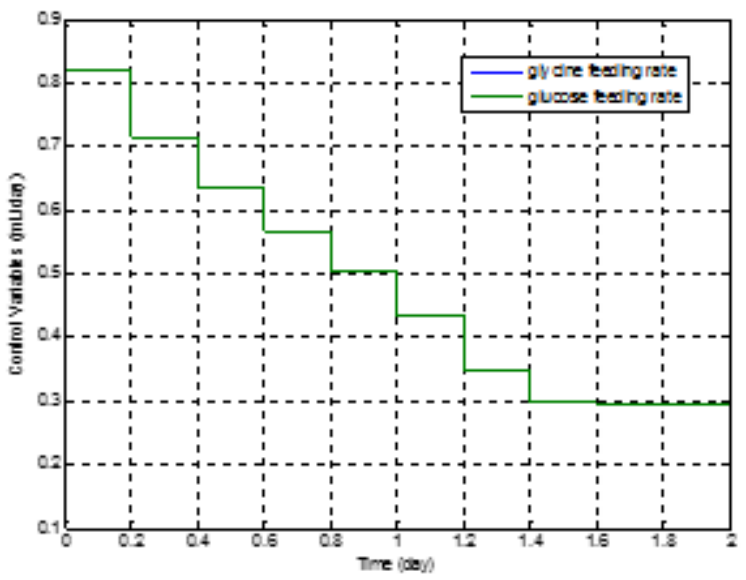

Fig. 3. Figure 3. Optimal feeding strategy of the microalgae growth model.

\section{REFERENCES}

[1] Y. Chisti, "Biodiesel from microalgae," Biotechnology Advances, vol. 25, no. 3, pp. 294-306, 2007.

[2] H. D. la Hoz Siegler, A. Ben-Zvi, R. Burrell, and W. McCaffrey, "The dynamics of heterotrophic algal cultures," Bioresource Technology, vol. 102, no. 10, pp. 5764-5774, 2011.

[3] H. D. la Hoz Siegler, W. McCaffrey, R. Burrell, and A. Ben-Zvi, "Optimization of microalgal productivity using an adaptive, non-linear model based strategy," Bioresource Technology, vol. 104, pp. 537-546, 2012.

[4] J. Abdollahi and S. Dubljevic, "Lipid production optimization and optimal control of heterotrophic microalgae fed-batch bioreactor," Chemical Engineering Science, vol. 84, pp. 619-627, 2012.

[5] D. S. Naidu, Optimal control systems. CRC press, 2002.

[6] T. Hirmajer, E. Balsa-Canto, and J. R. Banga, "Dotcvpsb, a software toolbox for dynamic optimization in systems biology," BMC Bioinformatics, vol. 10 , no. 1 , pp. $1-14,2009$. 